\title{
Surface adhesion of viruses and bacteria: Defend only and/ or vibrationally extinguish also?! A perspective
}

\author{
Manoj Kolel-Veetil ${ }^{1} \cdot$ Ayusman Sen ${ }^{2} \cdot$ Markus J. Buehler $^{3}$
}

Received: 20 January 2021 / Accepted: 28 May 2021 / Published online: 15 June 2021

(C) This is a U.S. government work and not under copyright protection in the U.S.; foreign copyright protection may apply 2021

\begin{abstract}
Coronaviruses COVID-19, SARS-CoV and NL63 use spikes in their corona to bind to angiotensin converting enzyme 2 (ACE2) sites on cytoskeletal membranes of host cells to deliver their viral payload. While groups such as disulfides in ACE2's zinc metallopeptidase, and also in COVID-19's spikes, facilitate such binding, it is worth exploring how similar complementary sites on materials such as polymers, metals, ceramics, fabrics, and biomaterials promote binding of viruses and bacteria and how they could be further engineered to prevent bioactivity, or to act as agents to collect viral payloads in filters or similar devices. In that vein, this article offers a perspective on novel tools and approaches for chemically and topologically modifying most utilitarian surfaces via defensive topological vibrational engineering to either prevent such adhesion or to enhance adhesion and elicit vibrational characteristics/'musical signatures' from the surfaces so that the structure of the binding sites of viruses and bacteria is permanently altered and/or their cellular machinery is permanently disabled by targeted chemical transformations.
\end{abstract}

\section{Perspective}

COVID-19 virus' startling impact on global citizenry has dramatically upended its quotidian existence in unimaginably manifold ways! As widely posited, the scale and speed of its impact might only have been matched previously by the Spanish Flu of 1918. Besides the existential threat that this virus poses to humanity, from a scientific point of view, it has spurred a collective global effort in the investigation and understanding of its insidious nature-with regard to its means of propagation, infection and affliction [1-5]. While vaccines and treatments will protect and treat the populace

Supplementary Information The online version contains supplementary material available at https://doi.org/10.1557/ s43580-021-00079-0.

Manoj Kolel-Veetil

manoj.kolel-veetil@nrl.navy.mil

1 Chemistry Division, US Naval Research Laboratory, Washington, DC 20375, USA

2 Departments of Chemistry and Chemical Engineering, Pennsylvania State University, University Park, PA 16802, USA

3 Laboratory for Atomistic and Molecular Mechanics, Massachusetts Institute of Technology, Cambridge, MA, USA from this virus, another aspect that needs an urgent, universal evaluation is an understanding of the mode and mechanism of transmission of this virus both via airborne and surface-facilitated means. In fact, there needs to be a universal effort in comprehensively understanding the ways by which viruses and bacteria transmit both in air and via surfaces as these types of calamities will only become more frequent owing to rapid globalization and the attendant unprecedented movement of the global population. Expectedly, such critical understanding should prevent the recurrence of disruption that the current COVID-19 crisis has inflicted on the globe. This Perspective is a proposed roadmap for establishing how both viruses and bacteria interact with utilitarian surfaces and how rather than only defend against such microorganisms as has been the norm, one may turn the table and give an existential fight to such invaders, responsible for both the current pandemic and possible future ones!

Specifically, in this Perspective, we attempt to understand the innate characteristics of viruses such as COVID-19 and bacteria that enable their adhesion to a variety of surfaces including plastics, metals, ceramics, fabrics and biomaterials. Admittedly, a lot of inspiration is drawn from the existing reported approaches that have mainly been employed for preventing bacterial and viral adhesion and infection [6-10]. In proposing a hypothesis, at the outset, it is important to reacquaint ourselves with the mostly taken for granted 
distinction that bacteria are 'living' systems due to their ability to produce energy for self-sustenance, and viruses are 'inert' as they need a host cell's biomachinery for their function and growth. Thus, the exact energy options that either bacteria or virus derives from inanimate materials such as plastics, metals, ceramics and fabrics and living materials such as skin and other biomaterials have to be understood fundamentally as it can lead to the development of appropriate methods for countering these organisms in each specific instance. Additionally, with viruses, while the structural alteration of their binding sites alone may be sufficient to achieve bio-inactivity, with bacteria, we may have to fundamentally disable them at a cellular level to render them ineffective. Further, the structural dimensions of viruses are in the $n m s$ and of bacteria in the $\mu \mathrm{ms}$ size regimes, respectively. Thus, even from a dimensional perspective they offer a broad spectrum of attendant (i.e. pertaining to their cores more so than their binding sites) molecular length scales to be considered during binding.

Coronaviruses, COVID-19 (Fig. 1), SARS-CoV and NL63, use spikes in their corona to bind to angiotensin converting enzyme2 (ACE2) sites on the cytoskeletal membranes of host cells to subsequently deliver their viral payloads $[11,12]$. Specific functionalities such as disulfide sites in both spike proteins and ACE2's zinc metallopeptidases facilitate such binding $[13,14]$. For most commonly encountered viruses and bacteria, there is considerable and reasonably solid understanding of the precise sites that facilitate such binding at the cellular level in humans. For example, the binding sites such as the spikes of a coronavirus and of

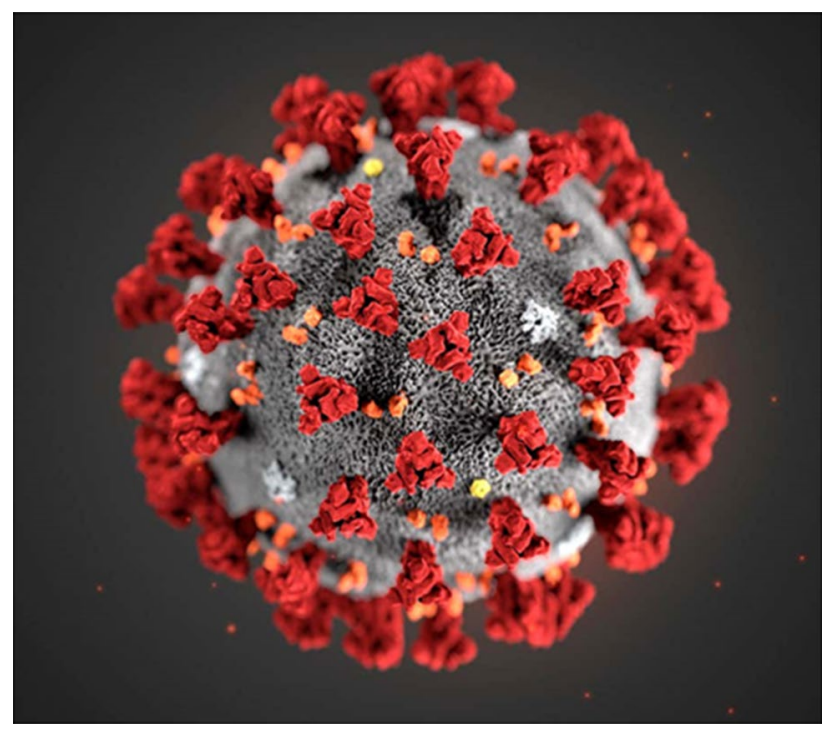

Fig. 1 The popular aesthetically pleasing depiction of the COVID19 virus; 2019-nCoV-CDC-23312.png. Reproduced with permission from the Centers for Disease Control and Prevention's Public Health Image Library (PHIL), ID\#23312 bacteria, and also of cytoskeletal sites of host organisms generally contain glycoprotein entities [15]. In contrast, data on the specific complementary binding sites used by viruses and bacteria for attachment to polymers, metals, ceramics and fabrics are sparse, as these inanimate objects are not typically closely scrutinized for their active constituent binding sites. The exceptions are studies that have taken inspiration from nature to produce biomimetic surfaces that reasonably mimic the surface characteristics of such natural systems [6-10]. This has been achieved by incorporating beneficial topological characteristics of biological systems such as surface roughness and wettability encompassing dispersive interactions and transient hydrogen bonding between such surfaces/entities and the microorganism to thwart its adhesion [16-18]. Importantly, all such strategies have been mainly driven by the sole desire to prevent adhesion of viruses and bacteria in the first place. While such strategies are admirable, ensuing repulsion, the viruses and bacteria are still alive and functional, i.e. they can still attach to hospitable sites to cause adhesion and infection at a future instance. Therefore, there is a need to devise new management strategies whereby in addition to preventing their adhesion, the vibrational signature of hospitable surfaces could be appropriately engineered with the aid of beneficial functional groups to facilitate a stronger adhesion of these microorganisms. Such adhered and surface-bound bioorganism ensembles may then be further manipulated by appropriate vibrational inputs to the surfaces leading to specific mechanobiological events in such microorganisms generating deleterious biochemical reactions leading to their permanent inactivity.

To further enable this vibrational modification strategy, one of us (Buehler) recently reported on the nanomechanical sonification of COVID-19's spike protein by a materiomusical approach [19-21] (see Supplementary File COVID19-sonification_excerpt.mp3 for an excerpt). Nanomechanical sonification is a method to use the nanomechanical vibrations of objects (e.g. atoms, molecules, assemblies of molecules) and use their spectrum of frequencies to generate an audible sound, using the music theoretic concept of transpositional equivalence. In other words, just like a macroscopic string generates an audible sound due to its mechanical vibrations that represent an overlay of set of fundamental sine waves, we can hear the characteristic sound, or timbre, of nanomechanical objects as we construct their sound. This is an exceptionally fertile platform that opens many avenues towards understanding of compatible vibrational signatures of these microorganisms and common utilitarian surfaces. In this vein, it should be obvious that every object in the Universe has its own innate vibrational signature. The need then is to create a library of innate vibrational signature of common utilitarian objects such as polymers, metals, ceramics and fabrics, using this materiomusical approach, 
to provide a reasonably exhaustive database of such material 'musical signatures'. Then, as Buehler suggested in his paper on the music of COVID-19, the intent would be to counter the innate vibrations of the interacting viruses and bacteria in a destructive way by providing counterpoints to these vibrations. In effect, by the use of defensive 'topological vibrational engineering (TVE)' to counteract these marauders! The concept of counterpoint, which in its own right is a cornerstone of classical music pioneered by JS Bach, provides a cross-cutting perspective that may help us understand the mechanics of intersecting points in structure within the protein (as executed in the musical translation by Buehler) but also enables the engineering of surface-viral interactions, especially to increase adhesion. In fact, counterpoint music - and its patterning of "note against note" is the penultimate interfacial musical genre that has moved human musical culture into a realm of intimate connectivity with mathematics, and other form and function in nature, as displayed in Bach's Goldberg Variations (Fig. 2) in brilliant ways [22]. With regard to surface-virus/bacteria interactions, such vibrational counterpoints should manifest as offensive vibrational inputs from the surface to the adhering microorganism.

Of course, engineering interfaces to trap viruses and bacteria, is easier said than done! So, how does one go about this? The creation of a vibrational musical library is a daunting task that cannot be achieved expeditiously without the assistance of some automated systems. Towards this, the application of artificial intelligence/machine learning (AI/ ML) approaches should be immensely helpful [23-25]. While from a fundamental sense, the application of Group Theory Principles [26], such as determining the point symmetry groups to arrive at reducible and irreducible representations of the vibrational modes to help delineate IR and Raman-active modes of these surfaces is logical, such analysis is extremely time consuming and also will be suitable only when there is periodicity and/or clearly defined disposition of bonds in the material such as in crystals and molecular compounds. In amorphous materials, that a majority of polymers and fibres are, such an analysis is not applicable and a more suitable approach would be to scan the surface for its molecular compositions to produce a 'mapping' of its vibrational signature structure. Understandably, the above task is not trivial and such expansive analysis will only be possible if one were to revert to utilizing AI/ ML approaches. AI/ML algorithms-enabled scanning of surfaces should generate vibrational signatures of each unique surface. Scanning of surfaces assisted with in operando surface scanning techniques such as Raman, IR or even XPS mapping that are coupled with such AI/ML algorithms [27] should be able to provide real time vibrational characteristics of surfaces so that appropriate surface modifiers may be produced onsite using advanced manufacturing techniques such as 3D and most beneficially, 4D printing techniques [28, 29]. Of course, while this may appear to be a utopian dream, it is quite likely that such advances may become a reality in due time. In addition to the innate vibrational characteristics, the vibrational signatures of surfaces may be further modified by using extraneously supplied frequencies that can produce additional 'counterpoints' on such surfaces.

While destructive 'counterpoints' can prevent the adhesion, could one make these counterpoints to be more conducive to binding? This opens up the fascinating possibility that such musical 'enticement' of the microorganisms
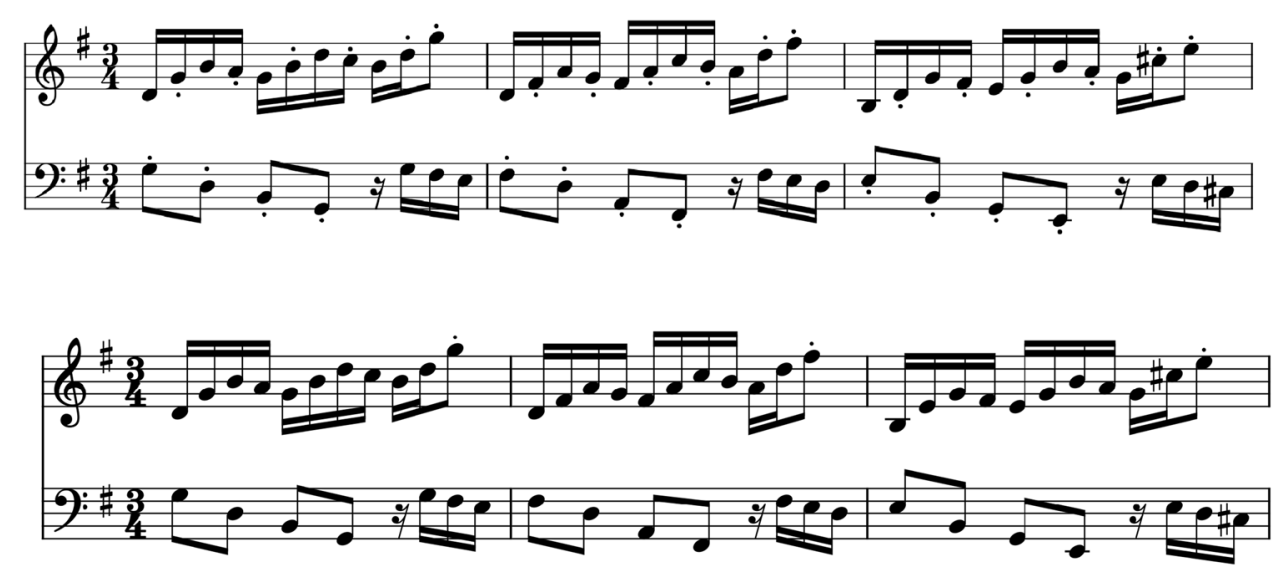

Fig. 2 Excerpt of Bach's Goldberg variations, showing "counterpoint"- two melodies played against each other (top and bottom, score created by M.J. Buehler based on the original composition by J.S. Bach, BWV 988, based on Variatia 8 a 2 Clav [22]. In musical composition, counterpoint is derived from the matching of multiple sequences or patterns, to form a conjoined musical expression where the whole is more than the sum of its parts, and where their interaction strength is derived from the level of intersectionality. This concept has analogy to the interaction of surfaces, or viruses and surfaces, or proteins with one another (see Supplementary File, Goldberg-Var 8.mp3) 
can lead to stronger and strategic binding of the microorganisms to further enable mechanobiological events that can lead to their permanent inactivity. In addition to an understanding of the vibrational modification, in pursuing TVE, the electronic changes that such engineering produces on a surface will have to be quantified in terms of the extent of the changes in the dispersion (van der Waals) (D), polar $(\mathrm{P})$ and hydrogen bonding $(\mathrm{H})$ characteristics of individual bonds and of the collective surface. An understanding of similar dispersion, polar and hydrogen bonding characteristics of the binding entity of a virus or a bacteria is also necessary and this can lead to such terms as $\Delta \mathrm{D}$, $\Delta \mathrm{P}$ and $\Delta \mathrm{H}$ which give an extent of differences in such properties between the two interacting species. From such values, the likelihood and extent of interaction between the TVE-modified surface and the microorganism can then be assessed based loosely on similar Hansen Solubility Parameter (HSP) evaluation of polymer-solvent solubility where the distance $(D)$ between the two species in the Hansen's parameter space is given by the following relation; $D^{2}=4\left(\delta D_{1}-\delta D_{2}\right)^{2}+\left(\delta P_{1}-\delta P_{2}\right)^{2}+\left(\delta H_{1}-\delta H_{2}\right)^{2}[30$, 31]. Of course, as in such HSP calculations, the smaller the $D$ value, the greater should be the binding interaction. Obviously, in our scenario, the polymer-solvent pair has to be replaced by the surface-microorganism pair.

With regard to TVE, an intriguing question that can be posed is whether one can separate such evolving musical frequencies of the surface into each of the three components, i.e. being due to specific contributions from changes in the forces of dispersion, polarity and hydrogen bonding of the initial entities on the surface. This would then enable a beneficial decoding of such ensemble vibrational milieu. Such an enhanced understanding would also provide us a means to controllably impact the innate bonding characteristics of a surface to effect and derive custom-made frequency compositions by the appropriate TVE inputs.
In assessing the electronic changes with TVE, one aspect to be considered is whether such changes can produce mechanobiological changes in the binding domains of the virus or bacteria. For example, if such binding can generate piezoelectric effects in the virus or bacteria, they can be tuned to impact the structure and consequently the efficacy of the binding entity of the microorganism such as spikes of COVID-19. One of us (Kolel-Veetil) recently reported on cyclic $\beta$-peptide amino acid structures, in which, due to the nature of the $\mathrm{C}=\mathrm{O}$ and $\mathrm{N}-\mathrm{H}$ entities of amide bonds being in specific directions normal to the cyclic plane, there is a resultant summation of the dipole moments that can lead to piezoelectricity $[32,33]$. Similar piezoelectricity-generating constructs should be quite general in our bodies as humans have evolved into energy-efficient systems that can function with much reduced amount of energy input to perform an extraordinary number of metabolic, neural and physical functions. In a recent review on such piezoelectric effects in various peptide and metabolite structures, the disposition of various dipole moments and piezoelectric potential in peptide nanotubes was elegantly described (Fig. 3) [34]. In fact, as early as in the 1970s, a report appeared on the piezoelectricity effects in myosin and actin [35] and more recently there have been reports of piezo- and flexoelectric membrane materials that control the action of fast biological motors in the ear [36]. While upon attachment to surfaces, such piezo effects could be exploited by viruses to 'kick start' some of their dormant cellular mechanism, one could also exploit such scenarios to counteract the viruses. Thus, by TVE of the surfaces one could facilitate appropriate adhesion scenarios to 'permanently alter the binding sites' of viruses and bacteria by disruption of their tertiary and quarternary protein structures so that on further contact of such surface-adhered species by humans and other species, they effectively remain innocuous! Such piezoelectricity-enabled effect is akin to modifying the hydrogen bonded structure of

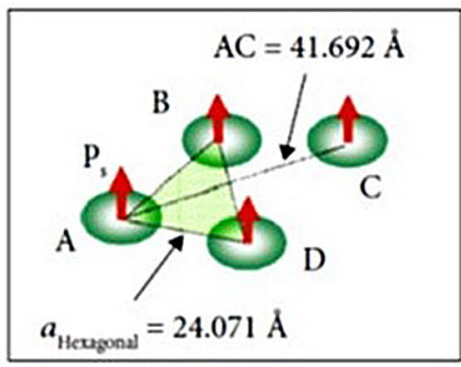

(a)

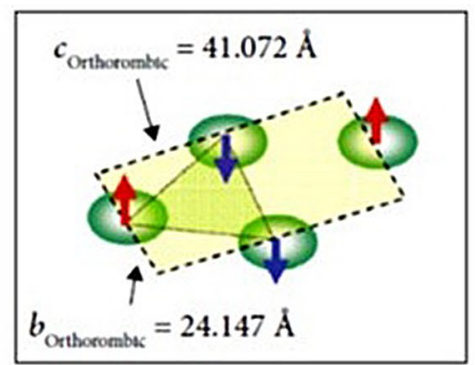

(b)

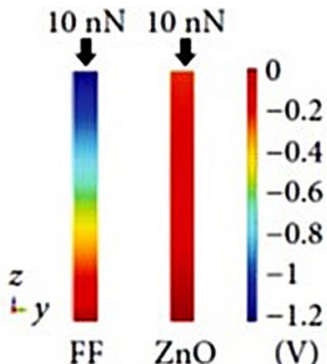

(c)

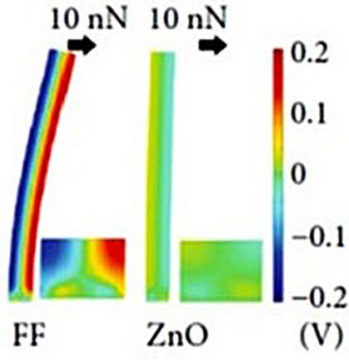

(d)
Fig. 3 Dipole moment distribution models of the hexagonal (a) and orthorhombic (b) structures of FF peptide nanotubes. c and d Simulation of electric potential in nanowires $500 \mathrm{~nm}$ long and $50 \mathrm{~nm}$ in diameter. c Piezoelectric potential in a $y-z$ plane slice for FF peptide (left) and $\mathrm{ZnO}$ (right) nanowires under $10 \mathrm{nN}$ of compressive force. d Piezoelectric potential in a $y-z$ plane for slice FF peptide (left) and $\mathrm{ZnO}$ (right) nanowires under $100 \mathrm{nN}$ transverse force. Insets show regions of potential reversal at the nanowire roots. Reproduced from Ref. [34] with permission from the American Association for the Advancement of Science 
the binding domains and other proximal domains in viruses and bacteria such as effected by pharmaceutical drugs Cobicistat, Ritonavir, Lopinavir, and Darunavir, all peptide-like, small molecules used currently for COVID-19 treatment that disrupt the hydrogen bonded tertiary and quarternary structures of the virus' spike proteins [37]. Also, with bacteria, one could think of utilizing such interactions to either augment or diminish their innate energy production capability by detrimentally impacting chemical cascades in the bacteria or virus. Thus, this is an exciting research area that require further exploration.

In thinking about impacting the biochemical cascades in the cellular machinery of bacteria and viruses, it is instructive to note that cells, i.e. human, bacterial and others, contain on the order of $300-400 \mathrm{mg} / \mathrm{mL}$ of proteins and in excess of 7000 different enzymes [38, 39]. In fact, $40 \%$ of all proteins in humans, bacteria, etc. use metals for their functions. This results in hundreds of major metabolic pathways, many of which are enabled specifically by transition metals. Some of the main transition metals and main group metals found in bacterial cells are $\mathrm{Mn}, \mathrm{Mg}, \mathrm{Cu}, \mathrm{Fe}$ and $\mathrm{Zn}$ $[38,39]$. At high amounts, metals are known to kill bacteria. Copper has been found to be especially toxic to bacteria at high concentrations [40, 41]. Hence, since time immemorial, $\mathrm{Cu}$ has been used as an antimicrobial including in pots to preserves food/drinks and as antifungal spray on vegetables. More recently, copper has been used extensively to treat and contain hospital-acquired infection and it is known to function both in aerobic and anaerobic conditions. Copper functions in both its reduced $\left(\mathrm{Cu}^{+1}\right)$ and oxidized $\left(\mathrm{Cu}^{+2}\right)$ forms giving it an ability to function in a broad range of conditions. While the above is a commentary only on copper, the same can be said of other transition metals and main group metals that are found in bacteria and viruses. In applying TVE, the electronic characteristics of the specific oxidation states of transition metals and main group metals with their distinct ligand fields may be perturbed by calibrated application of mechanical stimuli producing subtle changes in metal-ligand atom distances. This will be especially relevant and applicable to enzymatic systems involving such metals that are present in the cellular membranes of the microorganisms. This can in turn be expected to produce subtle changes in the energy levels of the bonding and non-bonding orbitals and hence, in the associated intra-metal and metal-to-ligand electronic transitions that occur and drive many of the redox processes during the enzymatic processes involving such metals. In general, the above hypothesis has its underpinning in the science of spin crossover complexes that are capable of modifications of the spin states of their metal sites upon an external stimulus such as temperature or pressure [42].

With regard to metal enzymes, mismetallation of enzymes is a highly effective approach that may be effected by the mechanobiological events produced by TVE [43]. For example, the biochemical mechanism in cells has evolved so that perfectly balanced thermodynamic criteria facilitate the uptake of the correct metal ion/s from the available metals pool for each enzyme present in the cells. However, a disruption of this finely balanced thermodynamical equilibrium can cause mismetallation such as, for example, in the bacterium Escherichia coli, superoxide and hydrogen peroxide can disrupt the equilibrium by oxidizing ferrous ion, thereby causing it to be displaced from many iron-dependent mononuclear enzymes [44]. Zinc or manganese ions are seen to replace such vacant sites conferring little activity and consequently producing metabolic logjams. One could envision such disruption of thermodynamic equilibria in bacteria and viruses that may be enabled by TVE to cause major disruption in such phenomena. While oxidative stresses to cells accumulate typically over a period of time and cause these disruptions, TVE can be used as a tool to produce such disruptions at a much faster time scale.

In the above vein, and with regard to other biochemical transformations that may be imposed in bacteria and viruses, one of us (Sen) has published extensively on the effects of enzymatic reactions and how mechanical energy is produced from biochemical reactions and how such energy may be channelled to direct the motion of enzymes up a concentration gradient of substrate; i.e. essentially chemotaxis at the molecular level, led and caused by transduction of chemical energy into mechanical motion [45-53]. The reverse should also be true wherein mechanical forces such as applied stresses/forces could alter enzyme-driven signalling pathways (Fig. 4). The ability of cells and cell components to move in response to chemical signals is vital for the survival of living systems. Specific enzymes can produce unique chemotactic response in response to an imposed substrate concentration gradient. While in healthy living cells, enzyme-driven motion is towards the substrate (energy source), with surface-bound bacteria and viruses, which become controlled by mechanical stimuli from TVE, one should be able to engineer such motion steered from hospitable sites wherein due to the presence of a non-essential element one could cause mismetallation and enzyme deactivation leading ultimately to the death of the microorganism.

Bacteria are known to produce autoagglutination of infected cells [54]. Autoagglutination is a reaction wherein suspended particles are aggregated or clumped. One of the most common agglutination reaction effected by bacterial infectivity is the agglutination of red blood cells in blood vessels which prevents the circulation of blood to various organs resulting in their failure [55]. The enzymatic metal sites provided by the bacteria accelerates the agglutination of proteins. This is seen to be one of the reasons why persons with blood group such as $\mathrm{A}$ and $\mathrm{AB}$ are more susceptible to COVID-19 than ones with $\mathrm{O}$ group, since the platelets in the former groups are favourably disposed to be agglomerated 


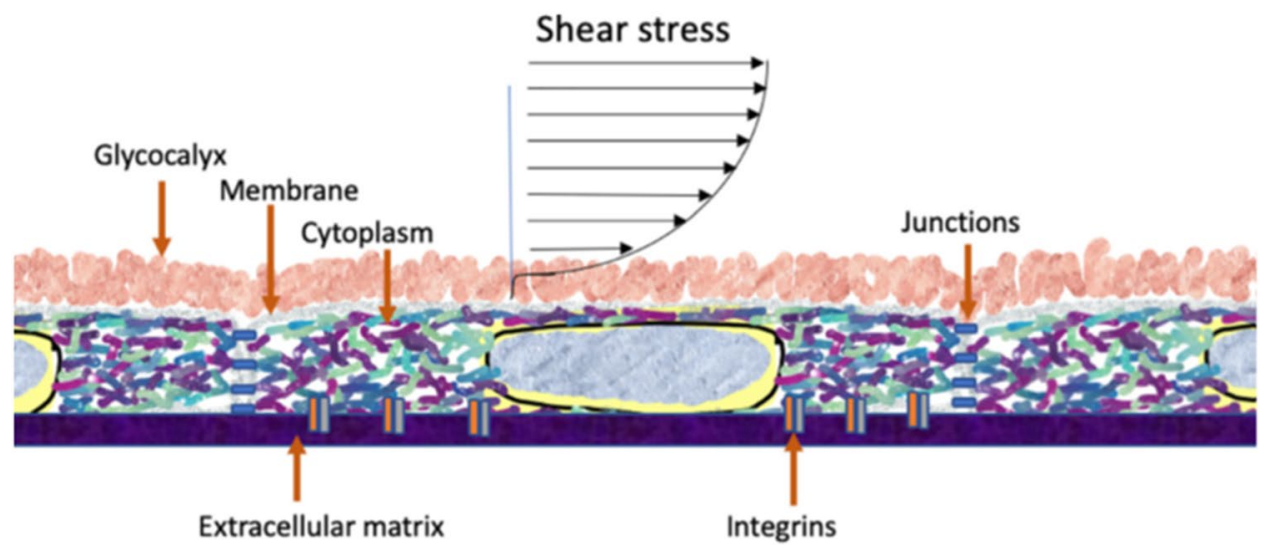

Fig. 4 A schematic of the shear stress of vascular endothelium, i.e. of blood vessels. The crowded glycocalyx, membrane and cytoplasm have inherently dynamic structures that are sensitive to the prevailing shear stress. Such structures are also arenas for enzyme-driven signal-

by active metal sites on COVID-19 [56]. If a similar reaction can be effected by surfaces on a contacting bacterium or virus, i.e. if the surfaces can induce 'clumping' of cellular materials in a bacterium, then the biochemical function of the bacterium can be permanently arrested leading to its death. This can be achieved by the adhering surface, upon binding by the bacteria, injecting adverse metal ions into their cellular machinery to cause such agglutination or agglomeration. Such surface-mediated interactions can be initiated by and should also be controllable by TVE.

Another aspect where topological transformations produced by TVE can cause disruption of cellular events in bacteria and viruses is by the mechanical forces that such interactions may impose on the cystoskeletons of the adhered bioorganism. Curvature-inducing effects of mechanical interaction are beginning to be understood more fundamentally in causing cancers, etc. In fact, the ability of proteins to sense membrane curvature is known to be essential to cellular function [57]. Also, a fine balance of such membrane curvature values has been known to maintain the equilibrium in production of exosomes and microvesicles formation and a disruption of such balance can lead to cancer in human cells. Furthermore, such membrane curvature and local pressure variations can also lead to agglutination reactions when a bacteria infects a human cytoskeletal membrane. Admittedly, even though this happens at a long temporal scale, the mechanical forces in action in such scenarios are also very low. In the TVE scenario, one could think of increasing the extent of these forces to such an extent so as to cause significant curvature effects on the cytoskeletons of bacteria and viruses. Such membrane curvature induction and local pressure generation could be engineered to be initiated by TVE and the viruses and bacteria can be thus be positioned to be at the receiving end! ling pathways, the location and kinetics of which are also dependent on applied force. Similar scenarios can be thought of in operation in most living cells. Reproduced from Ref. [53] with permission from the American Institute of Physics

\section{Concluding thoughts}

As experimental methods to measure vibrational signature in living organisms - small and large-improve, and with the advent of advanced computational methods such as ML/AI, we are going to be better positioned than ever to exploit holistic data from biology-spanning images to sound to other temporal variations-into a diagnostic and theranostic tool where we can begin to influence the processes of life and disease using alternate methods, going beyond traditional chemical drugs. In fact, we view that speaking the "language of life" with all its diverse manifestations offers opportunities for future drug designs that work with nature, not against nature, in our quest for a healthier and more sustainable planet that offers a harmonic and synergistic coexistence between species and a thriving human civilization.

Acknowledgments MB acknowledges financial support of this work by the IBM-MIT Watson AI Lab, the Army Research Office (W911NF1920098), the Office of Naval Research (N000141612333, N00014171-2320 and N000142012189), and AFOSR-MURI (FA955015-1-0514). Additional support from NIH is acknowledged (U01 EB014976). AS gratefully acknowledges funding by the Air Force Office of Scientific Research (FA9550-20-1-0393).

\section{Declarations}

Conflict of interest On behalf of all authors, the corresponding author states that there is no conflict of interest. 


\section{References}

1. D. Wrapp, N. Wang, K.S. Corbett, J.A. Goldsmith, C.-L. Hsieh, O. Abiona, B.S. Graham, J.S. McLellan, Science 367, 1260$1263(2020)$

2. Z. Sun, K. Thilakavathy, S.S. Kumar, G. He, S.V. Liu, Int. J. Environ. Res Public Health 17, 1633 (2020)

3. S. Laha, J. Chakraborty, S. Das, S.K. Manna, S. Biswas, R. Chaterjee, Infect. Genet. Evol. 85, 104445 (2020)

4. M.A. Shereen, S. Khan, A. Kazmi, N. Bashir, R. Siddique, J. Adv. Res. 24, 91-98 (2020)

5. C.M. Dasari, R. Bhuyka, Infect. Genet. Evol. 85, 104432 (2020)

6. R.J.V. Michael, B. Sambandam, T. Muthukumar, M.J. Umapathy, P.T. Manoharan, Phys. Chem. Chem. Phys. 16, 8541-8555 (2014)

7. S.L. Arias, J. Devorkin, J.C. Spear, A. Civantos, J.P. Allain, ACS Appl. Biol. Mater. 3(11), 7974-7988 (2020)

8. A. Tripathy, P. Sen, B. Su, W.H. Briscoe, Adv. Colloid Interface Sci. 248, 85-104 (2017)

9. Z. Jia, P. Xiu, M. Li, X. Xu, Y. Shi, Y. Cheng, S. Wei, Y. Zheng, T. Xi, H. Cai, Z. Liu, Biomaterials 75, 203-222 (2016)

10. P. Zhang, L. Lin, D. Zang, X. Guo, M. Liu, Small 13, 1503334 (2017)

11. A.J. Turner, J.A. Hiscox, N.M. Hooper, Trends Pharmacol. Sci. 25(6), 291-294 (2004)

12. J. Luan, Y. Lu, X. Jin, L. Zhang, Biochem. Biophys. Res. Commun. 526, 165-169 (2020)

13. S. Hati, S. Bhattacharyya, ACS Omega 5, 16292-16298 (2020)

14. S.D. Flora, R. Balansky, S.L. Maestra, FASEB J. 34, 1318513193 (2020)

15. A.C. Walls, Y.-J. Park, A. Tortorici, A. Wall, A.T. McGuire, D. Vessler, Cell 180, 281-292 (2020)

16. A. Lu, Y. Gao, T. Jin, X. Luo, Q. Zeng, Z. Shang, Ceram. Int. 46, 6550-6559 (2020)

17. X.-Q. Dou, D. Zhang, C. Feng, L. Jiang, ACS Nano 9(11), 10664$10672(2015)$

18. F. Lin, Z. Wang, J. Chen, B. Lu, L. Tang, X. Chen, C. Lin, B. Huang, H. Zeng, Y. Chen, J. Mater. Chem. B 8, 4002-4015 (2020)

19. M.J. Buehler, arXiv.org, e-Print Archive, Physics, 1-19 (2020)

20. C.-H. Yu, Z. Qin, F.J. Martin-Martinez, M.J. Buehler, ACS Nano 13(7), 7471-7482 (2019)

21. S. Franjou, M. Milazzo, C.-H. Yu, M.J. Buehler, Nano Futures 5(1), 012501 (2021)

22. D.J. Grossman, The Goldberg Variations - BWV 988, http://www. jsbach.net/midi/midi_goldbergvariations.html (1997)

23. K.T. Butler, D.W. Davies, H. Cartwright, O. Isayev, A. Walsh, Nature 559, 547-555 (2018)

24. J.G. Kim, S. Nozawa, H. Kim, E.H. Choi, T. Sato, T.W. Kim, K.H. Kim, H. Ki, J. Kim, M. Choi, Y. Lee, J. Heo, K.Y. Oang, K. Ichiyanagi, R. Fukaya et al., Nature 582, 520-524 (2020)

25. M. Gastegger, J. Behler, P. Marquetand, Chem. Sci. 8, 6924-6935 (2017)

26. F.A. Cotton, Chemical Applications of Group Theory, 3rd edn. (Wiley, New York, 1990)

27. N. Westing, J. Clark, D. Hooper, P. Nikolaev, B. Maruyama, MRS Online Proceedings Library, 1700 (Nanotubes and Related Structures-2014), 1-6 (2014)

28. X. Kuang, D.J. Roach, J. Wu, C.M. Hamel, Z. Ding, T. Wang, M.L. Dunn, H.J. Qi, Adv. Funct. Mater. 29, 1805290 (2019)

29. F. Momeni, S.M.M. Hassani, X. Liu, J. Ni, Mater. Des. 122, 42-79 (2017)
30. D.P. Faasen, A. Jarray, H.J.W. Zandvliet, E.S. Kooij, W. Kwiecinski, J. Colloid Interface Sci. 575, 326-336 (2020)

31. E. Spruijt, P.M. Biesheuvel, W.M.D. Vos, Phys. Rev. E 91(1), $012601(2015)$

32. K.P. Fears, M.K. Kolel-Veetil, D.E. Barlow, N. Bernstein, C.R. So, K.J. Wahl, X. Li, J.L. Kulp, R.A. Latour, T.D. Clark, Nat. Commun. 9, 4090 (2018)

33. M.K. Kolel-Veetil, LCDR L. Estrella, C.R. So, K.P. Fears, MRS Adv. 4(46-47), 2527-2532 (2019)

34. H. Yuan, P. Han, K. Tao, S. Liu, E. Gazit, R. Yang, AAAS 2019, 9025939 (2019)

35. H. Ueda, E. Fakuda, Jpn. J. Appl. Phys. 10(11), 1650-1651 (1971)

36. K.D. Breneman, R.D. Rabbitt, Materials Society Symposia Proceedings, Materials Research Society, 1186E (2009)

37. A. Blasiak, J.J. Lim, S.G.K. Seah, T. Kee, A. Remus, D.E. Chye, P.S. Wong, L. Hooi, A.T.L. Truong, N. Le, C.E.Z. Chan, R. Desai, X. Ding, B.J. Hanson, E. K-H. Chow, D. Ho, MedRXiv (2020) https://doi.org/10.1101/2020.05.04.20088104v1.full.pdf

38. L.D. Palmer, E.P. Skaar, Annu. Rev. Genet. 50, 67-91 (2016)

39. C.E. Flynn, S.-W. Lee, B.R. Peelle, A.M. Belcher, Acta Mater. 51, 5867-5880 (2003)

40. C.L. Dupont, G. Grass, C. Rensing, Metallomics 3, 1109-1118 (2011)

41. C.E. Santo, E.W. Lam, C.E. Elowsky, D. Quaranta, D.W. Domaille, C.J. Chang, G. Grass, Appl. Environ. Microbiol. 77(3), 794-802 (2011)

42. J.A. Real, A.B. Gaspar, M.C. Munoz, Dalton Trans. 2062-2069 (2005)

43. M. Huang, M.J. Parker, J.A. Stubbe, J. Biol. Chem. 289(41), 28104-28111 (2014)

44. J.A. Imlay, J. Biol. Chem. 289(41), 28121-28128 (2014)

45. K.K. Dey, S. Das, M.F. Poyton, S. Sengupta, P.J. Butler, P.S. Cremer, A. Sen, ACS Nano 8(12), 11941-11949 (2014)

46. K.K. Dey, X. Zhao, B.M. Tansi, W.J. Mendez-Ortiz, U.M. Cordova-Figueroa, R. Golestanian, A. Sen, Nano Lett. 15, 8311-8315 (2015)

47. P.J. Butler, K.K. Dey, A. Sen, Cell. Mol. Bioeng. 8(1), 106-118 (2015)

48. K.K. Dey, A. Sen, J. Am. Chem. Soc. 139, 7666-7676 (2017)

49. X. Zhao, H. Palacci, V. Yadav, M.M. Spiering, M.K. Gilson, P.J. Butler, H. Hess, S.J. Benkovis, A. Sen, Nat. Chem. 10, 311-317 (2018)

50. X. Zhao, K. Gentile, F. Mohajerani, A. Sen, Acc. Chem. Res. 51, 2373-2381 (2018)

51. A. Somasundar, S. Ghosh, F. Moharejani, L.N. Massenburg, T. Yang, P.S. Cremer, D. Velegol, A. Sen, Nat. Nanotechnol. 14, 1129-1134 (2019)

52. S. Ghosh, F. Mohajerani, S. Son, D. Velegol, P.J. Butler, A. Sen, Nano Lett. 19, 6019-6026 (2019)

53. P.J. Butler, APL Bioeng. 4, 010907 (2020)

54. D. Pulido, J. Arranz-Trullen, G. Prats-Ejarque, D. Velazquez, M. Torrent, M. Moussaoui, E. Boix, Int. J. Mol. Sci. 17, 552 (2016)

55. H. Fernandes, A. Fontes, A. de Thomaz, L. Barbosa, M. BarjasCastro, C.L. Cesar, Proc. SPIE 6644, 66440M (2007)

56. M.B. Barnkob, A. Pottegard, H. Stovring, T.M. Haunstrup, K. Homburg, R. Larsen, M.B. Hansen et al., Blood Adv. 4(20), 4990-4993 (2020)

57. W.F. Zeno, Baul, W.T. Snead, A.C.M. DeGroot, L. Wang, E.M. Lafer, D. Thirumalai, J.C. Stachowiak, Nat. Commun. 9, 4152 (2018) 